\title{
DETERMINISTIC CHAOS IN BLOOD PRESSURE SIGNALS DURING DIFFERENT PHYSIOLOGICAL CONDITIONS
}

\author{
Oliver P. Kinnane ${ }^{1}$, John V. Ringwood ${ }^{1}$, Rohit Ramchandra ${ }^{2}$, Carolyn J. Barrett ${ }^{2}$, \\ Sarah-Jane Guild ${ }^{2}$, Bridgit L. Leonard ${ }^{2}$ and Simon C. Malpas ${ }^{2}$ \\ ${ }^{I}$ Department of Electronic Engineering, National University of Ireland, Maynooth, \\ County Kildare, Ireland. \\ ${ }^{2}$ Circulatory Control Labroratory, Department of Physiology, University of Auckland, \\ Auckland, New Zealand.
}

\begin{abstract}
Several coupled and nonlinear controlling mechanisms are involved in the regulation of blood pressure. The possible presence of chaos in physiological signals has been the subject of some research. In this study, blood pressure signals were analysed using a range of nonlinear time series analysis techniques. Individual effectors of blood pressure were either experimentally removed or enhanced, so that the controlling mechanisms that are responsible for the chaotic nature of the signals may be identified by chaotic analysis of the signals. The level of chaos varied across the different experimental conditions, showing a distinct decrease from control conditions to all other experimental conditions. Copyright (C) 2003 IFAC
\end{abstract}

Keywords: Biomedical systems; Chaos theory; Lyapunov; Pressure; Nonlinear analysis

\section{INTRODUCTION}

The mechanisms that control cardiovascular function have long been accepted as highly complex and nonlinear systems. The baroreflex mechanism, through the control of the action of the heart and vasculature, is chiefly responsible for the regulation of blood pressure (BP) in the short term. Other paracrine and hormonal mechanisms, including those that involve nitric oxide (NO) and angiotensin II are also intimately involved in the maintenance of BP within certain operating bounds. Both the interrelationships of these regulatory mechanisms and the inherent nonlinearities of each individual mechanism, result in the complex and nonlinear behaviour of the BP controlling system.

The use of linear time and frequency domain techniques have proven to be powerful tools for the analysis of BP signals. However, there is a paucity of studies that use nonlinear time series analysis techniques. A branch of these techniques, extends to the detection and quantification of chaos which manifests itself in nonlinear systems.

The broader work of these researchers is rooted in the modelling of the nonlinear mechanisms that control BP. Hence, this chaotic analysis of BP signals is an obvious extension of this research, and has the aim of identifying the nonlinear elements in the BP controlling mechanisms that are responsible for chaos in BP.

Prior to the investigation for chaos in the BP signals, nonlinear deterministic structure was detected using surrogate data techniques. Although, at times viewed with skepticism by researchers of chaos, the established chaotic analysis methods of correlation dimension and largest Lyapunov exponents (LLE) calculation were used to investigate for chaos in the signals, taking special care to accurately choose the embedding parameters and to minimise the effects of measurement noise on the signals. More recently developed methods of chaos detection were also investigated (Poon and Barahona, 2001) and preliminary results using this method support the results already attained.

The use of the chaotic analysis techniques revealed good evidence of chaos in BP signals. Furthermore removal of one of the principle short-term controllers of blood pressure, the arterial baroreflex loop, shows a decrease in the LLE, suggestive that under this condition, BP is more predictable, a result previously documented by Wagner et al (1995). An obvious question arising from this study is whether this change occurs when other BP controlling mechanisms are removed or affected. In other words, to what extent do the other effectors of BP (egs NO and angiotensin II) affect the level of chaos in BP?

In the present study we recorded BP in conscious rabbits during seven different experimental conditions. The level of chaos during each condition is quantified and conclusions regarding the role of the different effectors in the generation of chaos in $\mathrm{BP}$ are drawn. 
All experiments were performed by the physiologists of the Circulatory Control Laboratory at the University of Auckland, New Zealand. BP signals were recorded in conscious New Zealand white rabbits. All experiments were approved by the University of Auckland, Animal Ethics Committee. The signals were continuously recorded and sampled at $500 \mathrm{~Hz}$ using an analog-to-digital data acquisition card (National Instruments). Calibrated signals were continuously displayed on screen and saved using a program written in the LabVIEW graphical programming language (National Instruments).

Data was recorded during seven different physiological conditions:

1. Control rabbits. BP was measured via an implantable telemetry device inserted in a central ear artery of 10 resting and conscious rabbits (see: Leonard, et al., 2000; Ramchandra, et al., 2002).

2. Blood volume expansion. In 5 rabbits a polygeline-electrolyte solution was used to increase plasma volume. This solution was administered at $1.5 \mathrm{ml} / \mathrm{min} / \mathrm{kg}$ for $15 \mathrm{mins}$. (see: Leonard, et al., 2000).

3. Control after blood volume expansion. BP was recorded for a further 7 mins after completion of volume expansion. (see: Leonard, et al., 2000).

4. Baroreceptor denervated rabbits. The afferent nerves sensing BP were cut in a group of 6 rabbits. Recordings were taken from rabbits at least 10 days after surgery and with the animals living in their home cages and on a $12 \mathrm{hr}$ light/dark cycle.

5. Baroreceptor denervated rabbits with infused angiotensin II. Following barodenervation, the animals were infused with angiotensin II (50 $\mathrm{mg} / \mathrm{kg} / \mathrm{min})$.

6. Angiotensin II infused rabbits with intact baroreflex. These baroreflex animals were infused with angiotensin II (50 $\mathrm{mg} / \mathrm{kg} / \mathrm{min})$.

7. NO blocked rabbits with intact baroreflex. In a group of 4 rabbits, NO blocker L-NAME was induced by oral administration and the daily intake was $50 \pm 4 \mathrm{mg} / \mathrm{kg} / \mathrm{day}$ (Ramchandra, et al., 2002).

Analysis of variance statistical techniques were applied (ANOVA). P values below 0.05 were considered significant. All values are presented as mean \pm standard deviation (S.D.).

\section{METHODS OF NONLINEAR TIME SERIES ANALYSIS}

The investigation for chaos in the experimentally recorded blood pressure signals begins with the reconstruction of the phase space.

For an $\mathrm{N}$-point time series, $x(i)=\left\{x_{1}\right.$, $\left.x_{2}, \ldots \ldots \ldots \ldots, x_{N}\right\}$, each time-delay vector in phase space is formed as:

$$
X_{i}=\left[x_{i}, x_{i+\tau}, \ldots \ldots \ldots \ldots, x_{i+(m-1) \tau}\right]
$$

where $\tau$ is the time delay, $m$ is the minimum embedding dimension. These vectors form the rows of the matrix $\mathbf{X}$, which describes the reconstructed phase-space.

$$
\mathbf{X}=\left[X_{1}, X_{2}, \ldots \ldots \ldots X_{M}\right]^{T}
$$

where, $M=N-(m-1) \tau$.

The time delay was calculated using the automutual information method of Fraser and Swinney (1986). This method gives a measure of the general dependence between the elements of the observed time series. The use of the autocorrelation method for calculating the time delay was also investigated, however, a major limitation of this method is that the results are a measure of linear dependence, so it is not the most appropriate method to analyse the time series of a variable of a nonlinear system.

The embedding dimension was calculated using the method proposed by Cao (1997). Although a number of other algorithms exist for calculating the embedding dimension, the method of Cao (1997) attains much acclaim in the literature as it overcomes the disadvantages of the other algorithms, which require a large number of data points, are too subjective or computationally very intensive (Yeragani, et al., 2002).

\subsection{Nonlinear noise reduction}

The existence of noise is inherent in experimentally recorded physiological signals. This noise may completely obscure the underlying fractal structure unless the data are preprocessed to reduce it (Grassberger, et al., 1991). Considering that noise often manifests itself at higher frequencies, a common technique amongst researchers, when attempting to minimise the level of noise in an experimentally recorded signal, is to use linear lowpass filtering techniques. However, a defining feature of chaotic signals is that they will generally have a broad spectrum, with frequency components at all frequencies across the spectrum. Hence, any attempt to reduce noise by using a linear filter will have a detrimental effect on the signal resulting in major signal distortion. Also, physiological systems are generally accepted to be highly nonlinear, therefore the use of linear filtering techniques when dealing with signals recorded from such nonlinear systems is imprudent.

Alternatively, a number of nonlinear noise reduction algorithms exist, which avoid the distortion of the signal due to linear filtering. The algorithm chosen for this study is that proposed by Schreiber (1993), which, simply aims to replace each data point, $x_{i}$, by the average value of this coordinate over points in a suitably chosen neighbourhood. 
This algorithm was implemented so as to minimise the effect of measurement noise on other calculated values in this study.

\subsection{Surrogate data analysis}

Surrogate data is used to test for nonlinear deterministic structure. Surrogate data is artificially generated data that mimics certain linear features (autocorrelation function, spectral power density) of the specified time series but that is otherwise stochastic. The experimentally recorded time series and this surrogate data are then used to test a specific hypothesis. In this case a null hypothesis is chosen which, is best described as the default conclusion in the lack of contrary evidence (Theiler and Pricard, 1996). The null hypothesis in this case is, that the data arose from a linear random process.

In order to test the null hypothesis, a discriminating statistic is chosen. A significant difference between the discriminating statistic calculated for the original experimentally recorded data and for the surrogate data sets indicates the existence of nonlinear structure in the original data. In principle, any nonlinear statistic that assigns a real number to a time series can be chosen as the discriminating statistic (Kantz and Schreiber, 1997). The measures chosen for this study are a nonlinear prediction error, calculated using a simple nonlinear prediction algorithm (Kantz and Schreiber, 1997) and the LLE. The prediction error was chosen as nonlinear predictability provides a direct test for the presence of determinism in the data set (Sugihara and May, 1990) and the LLE because it is the quantifier which we are mainly interested in, in this study.

The technique used to create the surrogates is that proposed by Theiler et al (1996). Using this technique surrogate data is generated by randomising the phases of the Fourier transform of the time series.

\subsection{Correlation dimension}

The correlation dimension is calculated using the method of Grassberger and Procaccia (1983), where the correlation dimension is the slope of the plot of the correlation sum verses the radius of the neighbourhood chosen. The correlation sum is:

$$
C(\varepsilon)=\lim _{N \rightarrow \infty} \frac{1}{N^{2}} \sum_{i=1}^{N} \sum_{j=1}^{N} G\left(\varepsilon-\left|x_{i}-x_{j}\right|\right)
$$

and, where, $G$ is the Heaviside function, $G(y)=0$ if $y$ $\leq 0$ and $G(y)=1$ for $y>0$. The sum counts the pairs $\left(x_{i}, x_{j}\right)$ whose separation is smaller than $\varepsilon$. The slope of successive plots of the log of the correlation sum verses the log of the radius of neighbourhoods for increasing embedding dimension will stop changing as the optimum value of the embedding dimension is reached.
The correlation dimension is a very popular quantifier of chaos as it yields an approximate estimation of the fractal dimension. Although the term fractal dimension is used rather indiscriminately, it is often seen as a measure of the number of degrees of freedom, which comprise the dynamics of a system (Holstein-Rathlou and Marsh, 1994).

\subsection{Largest Lyapunov exponent}

A phase-space trajectory reflects the evolution of a dynamical system and the Lyapunov exponents enumerate the average rate of convergence or divergence of two neighbouring trajectories in phase space. A $m$-dimensional system has $m$ Lyapunov exponents, however, the presence of one positive exponent denotes chaos. A positive exponent implies the divergence of two trajectories and therefore, the value of the positive exponent quantifies the sensitivity of the system to initial conditions.

\subsection{Numerical titration}

A novel method for the detection of chaos, based on a numerical titration process, has been developed by Poon and Barahona (2001). This technique involves using additive white noise as a titrant for chaos. The method simply involves adding white noise of increasing standard deviation to the data until the nonlinearity in the data goes undetected by a certain chosen nonlinear indicator. The proposed candidates for this nonlinear indicator are a time-reversibility measure and the Volterra-Wiener nonlinear identification method.

\section{RESULTS}

The phase-space is reconstructed for the implementation of the nonlinear noise reduction algorithm using unity time delay and an embedding dimension, $m$, of 25 . These parameter values provided the optimum results for the algorithm chosen.

Kantz and Schreiber, (1997) propose that a good choice of the size of the neighbourhood, $U_{i}^{\varepsilon}$, is given by 2-3 times the noise amplitude. The data recordings are to one decimal place, therefore, it may be assumed that the discretisation error is distributed in the range $[-0.05,0.05]$. The radius was chosen to at least include this range of error, and was decreased exponentially during 3-5 iterations of the procedure. Delay maps of a BP time series before and after noise 
reduction are presented in Figure 1 and 2 .

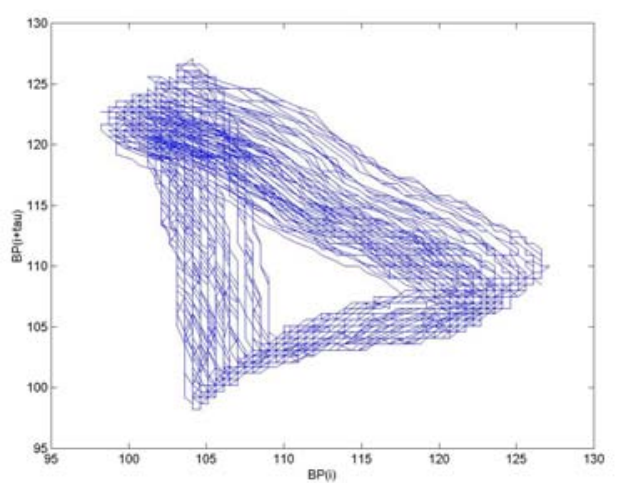

Fig. 1. Delay maps of 10,000 points of a blood pressure time series before noise reduction.

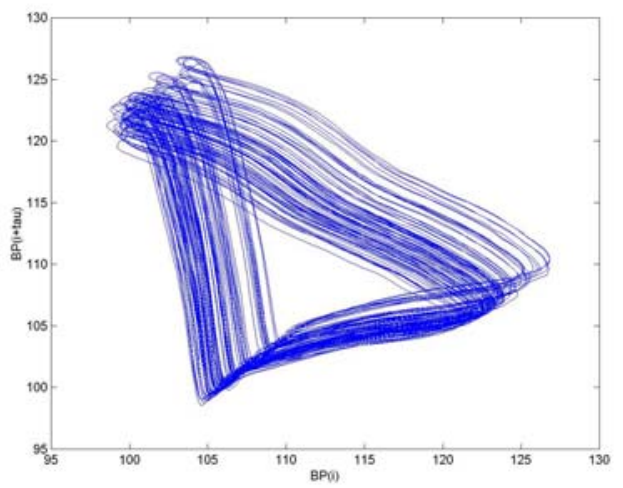

Fig. 2. Delay maps of 10,000 points of a blood pressure time series after noise reduction.

The delay maps resulted in a similar doughnut shape to those reported by other investigators of BP (Wagner, et al., 1995; Lovell, et al., 1997).

The phase-space of the, now noise reduced, time series is again reconstructed using the time delay embedding method, with a time delay of 40 samples and an embedding dimension of 10 . The embedding parameters are maintained at these values across all animals and physiological conditions. The plot of the correlation sum verses the radius supports 10 as a good choice of embedding dimension, as the slopes of the consecutive plots become constant at approximately this value of embedding dimension. An example of such a plot is given in figure 3 .

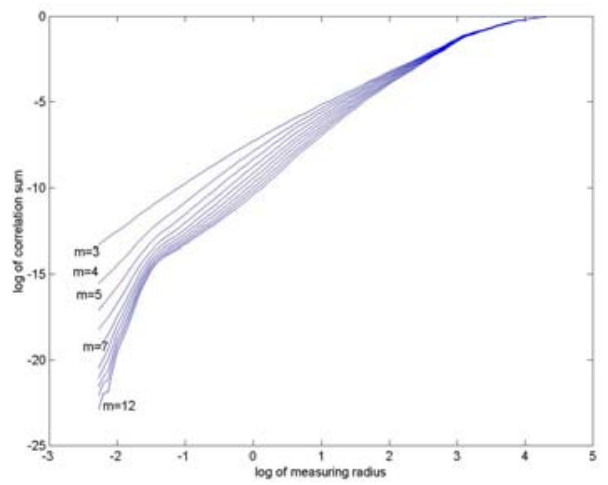

Fig. 3. Plot of correlation sum verses measuring radius for data of a single animal, as an example.
Twenty surrogate data sets were created for each time series. Both the LLE and the nonlinear prediction error were used as discriminating statistics The null hypothesis stated that the time series originate from a linear process. This null hypothesis is rejected as both discriminating statistics are significantly different from the original time series to all surrogate data sets $(\mathrm{P}<0.05)$. The prediction error was calculated for 1 to 100 time steps ahead for each time series and the forecasting error in the experimental time series was less than that for the surrogate data sets at each prediction. The values of the LLE calculated for the surrogate data sets, although significantly decreased from the LLE for the original time series were still positive.

The LLE for the control subjects was significantly higher than all but one of the other experimental conditions $(\mathrm{P}<0.01)$. This exceptional case is for the rabbits in the NO blocked experimental condition. The average values of the LLE, along with the average correlation dimension, for each experimental condition are documented in table 1 . All values are presented as mean \pm S.D.

The correlation dimension varied greatly across animals, hence, the average values of the correlation dimension calculated for the different experimental conditions are generally not statistically different $(\mathrm{P}>0.05)$ from one another, making it unfeasible to draw conclusions.

\section{TECHNICAL LIMITATIONS}

The choice of the embedding parameters for the reconstruction of the phase-space was one that had to be made with care due to the considerable sensitivity of the LLE to variations in these values. This choice is always going to be approximate and subjective, however, care was taken when calculating these values and so as to enable accurate comparison, both the time delay and embedding dimension were maintained constant for all data sets and physiological conditions. It may be argued that the structure of the system may change during barodenervation or NO blockade, however, there is not sufficient clarity in the data to suggest that this is the case and the tests for these embedding parameters did not present any palpable results to demonstrate this to be the case. Hence, it is assumed that the structure of the system remains the same but that the parameters of the system change.

With experimentally recorded data, problems and limitations are always to be expected due to errors during the recording, the restriction of the resolution of each data point and the masking of the data by noise. The use of nonlinear noise reduction minimises the problems created by the measurement noise. The restriction of the resolution of the data points is a limitation posed by the finite number of bits of the A/D converter. 


\begin{tabular}{cccc}
\hline $\begin{array}{c}\text { Experimental Condition } \\
\text { (Number of animals) }\end{array}$ & Mean BP & $\begin{array}{c}\text { Correlation } \\
\text { Dimension }\end{array}$ & $\begin{array}{c}\text { Largest Lyapunov } \\
\text { exponent (LLE) }\end{array}$ \\
\hline Control subjects (10) & $75 \pm 9$ & $2.7 \pm 0.3$ & $1.70 \pm 0.10$ \\
Baroreceptor denervated subjects (5) & $87 \pm 4$ & $2.4 \pm 0.2$ & $1.14 \pm 0.11$ \\
Baroreceptor denervated \& angiotensin II & $108 \pm 3$ & $2.6 \pm 0.1$ & $1.23 \pm 0.11$ \\
infused (5) & & & \\
Nitric oxide blocked subjects (5) & $84 \pm 3$ & $2.4 \pm 0.5$ & $1.61 \pm 0.05$ \\
Blood volume expansion (5) & $59 \pm 7$ & $3 \pm 1$ & $1.48 \pm 0.09$ \\
Control after blood volume expansion (5) & $75 \pm 15$ & $3.9 \pm 0.4$ & $1.24 \pm 0.22$ \\
Angiotensin II infused (3) & $104 \pm 8$ & $2.7 \pm 0.3$ & $1.33 \pm 0.06$ \\
\hline
\end{tabular}

Table 1 Averaged values of the LLE calculated for the seven different physiological conditions

There is no ideal solution to this problem, however, the effects of the bias due to quantization on the calculated values of the LLE are diminished by averaging over a large number of data points.

The value of the LLE for the stochastic surrogate data was positive. This is an unexpected result, however, a positive LLE was previously reported for purely stochastic data Damming and Mitschke (1993).

\section{DISCUSSION}

The main aim of this study was to test how the shortterm dynamics of BP control are affected when short to medium term effectors of blood pressure are either removed or enhanced. The results of the surrogate data tests demonstrate that the underlying controlling mechanisms of blood pressure are nonlinear. The positive LLEs of all the animal groups, by definition, indicate that these nonlinear mechanisms exhibit chaotic behaviour. Preliminary results using the numerical titration technique proposed by Poon and Barahona (2001) also support the findings of the presence of chaos in BP signals.

Documented in table 1 are the average LLE values calculated from BP signals recorded in animals during different experimental conditions. The positive values of the LLEs vary significantly from the control group to all but one of the other groups (the group of NO blocked animals), indicating a decrease in chaos from the control situation to these other situations.

The significant variation in the value of the LLE during the different experimental conditions leads to the conclusion that certain effectors of BP play more of a role than others in the establishment of chaos. The value of the LLE drops by over $30 \%$ from the control subjects to the barodenervated animals, i.e. from 1.7 to $1.14(\mathrm{P}<0.01)$. A similar result, which used dogs as subjects, was previously documented by Wagner, et al., (1995). When the animals are barodenervated the signal becomes less chaotic and more predictable.
This may be explained by the fact that after the removal of the baroreflex, the ability to control BP in the short-term is repressed. Wagner, et al. (1996) proposed that other effectors of BP will compensate this loss of control (Brand, et al., 1988), hence, perturbing the dynamics of the regulating system in a way that is simpler and less complex.

Infusion of the vasoconstrictive angiotensin II in baroreflex intact animals leads to an increase in the mean level of BP (from $75 \pm 9$ to $104 \pm 3 \mathrm{mmHg}$ ) and a decrease in the LLE (from 1.7 to 1.33 $(\mathrm{P}<0.05))$. This decrease in the value of the LLE may be related to the increase in the constrictive state of the blood vessel walls during angiotensin II infusion, which may reduce the ability of the short-term BP controlling mechanisms to react, hence an increase in the predictability of the BP signal and a decrease in chaos.

The LLE calculated for the NO blocked group of animals did not show a significant decrease from the control subjects. This result is surprising as NO is known to play a prominent role in the short-term control of BP (Rees, et al., 1989). Again compensatory effects may come into play, which in this case maintain the level of chaos close to that of the control values. Yuasa, et al., (2000) documented results that suggest an increase in sympathetic nervous activity, a pathway of the baroreflex, after NO inhibition. This increased activity of the baroreflex may help maintain the level of chaos in BP after NO inhibition.

Studies documenting the variation in the LLE of heart rate signals abound in the physiology literature. A large percentage of these studies document the decrease in the LLE from the healthy to the unhealthy condition such as depression (Yeragani, et al., 2002), MS (Ganz, et al., 1998) and epilepsy (Faustmann and Ganz, 1994). This broad assertion is reflected in the results of this study in which the LLE decreases from the control situation to all other situations, during which the controllers of BP are tampered with. These situations include the unhealthy conditions of a damaged baroreflex control mechanism and hypertension among others.

In this study we showed that perturbation of the overall BP controlling mechanism by removal or enhancement of some of the effectors of BP resulted 
in a decreased level of chaos in the recorded BP signals. It is the future aim of these researchers to develop a model of the BP controlling mechanisms and their interactions, and attempt to replicate and hence, better explain the results documented in this paper.

Acknowledgements: : We gratefully acknowledge the financial support of the Dean's fund, NUI Maynooth and the Wellcome Trust for their provision of a travel grant that has allowed successful collaboration between the Control systems group at NUI Maynooth and the Circulatory Control Labroratory, at the University of Auckland. Many thanks also to Prof. D. Heffernen of the Department of Mathematical Physics, NUI Maynooth for many stimulating discussions.

\section{REFERENCES}

Brand, P.H., P.J. Metting and S.L. Britton (1988). Support of arterial blood pressure by major pressor systems in conscious dogs. Am. J. Physiol., 255, H483-H491.

Cao, L. (1997). Practical method for determining the minimum embedding dimension of a scalar time series. Physica D., 110, 43-50.

Faustmann, P.M. and R.E. Ganz (1994). Central cardio autonomic disorganization in interictal states of epilepsy detected by phase space analysis. International Journal of Neuroscience, 78, 43-7.

Fraser, A.M. and H.L. Swinney (1986). Independent coordinates for strange attractors from mutual information. Phys. Rev. A., 33, 1134-1140.

Ganz, R.E., G. Weibels, K.H. Stacker, P.M. Faustmann and C.W. Zimmermann (1993) The Lyapunov exponents of heart rate dynamics as a sensitive marker of central autonomic organization: An exemplary study of early multiple sclerosis. Int. J. Neuroscience, 71, 2936.

Grassberger, P. and I. Procaccia (1983). Measuring the strangeness of a strange attractor. Physica $D$. Amsterdam, 9, 189-203.

Grassberger, P., T. Schreiber and C. Schaffrath. (1991). Non-linear time sequence analysis. Int $J$. Bifurcation and Chaos, 1, 521.

Lovell, N., B. Henry, A. Avolio, B. Celler, D. Carlson, and M. Brunner (1997). Nonlinear chaotic dynamics of mean arterial pressure after carotid baroreceptor isolation. In: Proceedings, $19^{\text {th }}$ International Conference - IEEE/EMBS, Chicago, USA, October 1997. IEEE.

Leonard, B.L., M.A. Navakatikyan and S.C. Malpas (2000). Differential regulation of the oscillations in sympathetic nerve activity and renal blood flow following volume expansion. Autonomic Neuroscience: Basic and Clinical, 83, 19-28.

Kantz, H. and T. Schreiber, (1997). In: Nonlinear Time series analysis. Cambridge, United Kingdom: Cambgidge University Press.

Poon, C.S. and C.K. Merrill (1997). Decrease in cardiac chaos in congestive heart failure. Nature, 389, 492-495.

Rosenstein, M., J.J. Collins and C.J. De Luca (1993). A practical method for calculating largest Lyapunov exponents from small data sets. Physica D., 65, 117-134.

Ramchandra, R., C.J. Barrett and S.C. Malpas (2002). Unpublished results.

Rees, D.D., R.M. Palmer and S. Moncada (1989). Role of endothelium-derived nitric oxide in the regulation of BP. Proc. Natl. Acad. Sci. USA., 86, 3375-8.

Schreiber, T. (1993). Extremely simple nonlinear noise reduction method. Phys Rev E, 47, 2401-4

Schreiber, T. and A. Schmitz (1996). Improved surrogate data for nonlinearity tests. Phys. Rev. Lett., 77, 635-9.

Sugihara, G. and R.M. May (1990). Nonlinear forecasting as a way of distinguishing chaos from measurement error in time series. Nature, 344, 734-741.

Theiler, J. S., Eubank, A. Longtin, B. Galdrikian and J. Doyle Farmer (1992). Testing for nonlinearity in time series: the method of surrogate data. Physica D., 58, 77-94.

Wagner, C.D., R. Mrowka, B. Nafz, and P.B. Persson, (1995). Complexity and "chaos" in BP after baroreceptor denervation of conscious dogs. Am. J. Physiol., 269, H1760-H1766.

Wagner, C.D., B. Nafz, and P.B. Persson, (1996). Chaos in blood pressure control. Cardiovascular research, 31, 380-387.

Yeragani, V.K., R. Rao, A. Jayaraman, R. Pohl R. Balon and D. Glitz (2002). Heart rate time series: decreased chaos after intravenous lactate and increased non-linearity after isoproteerenol in normal subjects. Psychiatry Research, 109, 81-92.

Yuasa, S, X. Li, H. Hitomi, M. Hashimoto, H. Fujioka, H. Kiyomoto, K. Uchilda, T. Shoji, N. Takahashi, S. Miki, A. Miyatake, K. Mizushige and H. Matsuo (2000). Sodium sensitivity and sympathetic nervous system in hypertension induced by long-term nitric oxide blockade in rats. Clin. Exp. Pharmacol. Physiol., 27, 18-24. 